\title{
Intravitreal Daptomycin for Recalcitrant Postoperative Endophthalmitis
}

\author{
Jennifer M. Sim Kapil G. Kapoor Alan L. Wagner \\ Wagner Macula and Retina Center, Eastern Virginia Medical School, \\ Virginia Beach, Va., USA
}

\section{Key Words}

Culture negative $\cdot$ Daptomycin · Endophthalmitis · Resistance $\cdot$ Retina $\cdot$ Vancomycin

\begin{abstract}
Purpose: To report the first case to our knowledge of intravitreal daptomycin used to successfully treat culture-negative vancomycin resistant to exogenous endophthalmitis. Methods: Case report with preoperative, intraoperative, and postoperative findings. Results: A 63year-old Caucasian male underwent routine pars plana vitrectomy with epiretinal membrane peeling. He developed acute postoperative endophthalmitis, and underwent vitreous tap and injection of intravitreal vancomycin/ceftazidime/dexamethasone. Gram stain showed Grampositive cocci, but cultures were negative. His infection subsequently proved very recalcitrant and his treatment course involved pars plana vitrectomy with anterior chamber washout and repeat injection of antibiotics, followed by repeat intravitreal vancomycin and ceftazidime. Ultimately, a second vitrectomy with intravitreal daptomycin controlled his intraocular infection. On each occasion, cultures were negative. Conclusion: This case suggests that vancomycin resistance should be considered in culture-negative postoperative endophthalmitis, and intravitreal daptomycin should be considered as an important treatment alternative. Although vancomycin resistance is fairly rare in endophthalmitis, acknowledgment of its increasing occurrence rate is critical for optimal management.

C 2016 The Author(s)

Published by S. Karger AG, Basel
\end{abstract}

\section{Introduction}

Endophthalmitis is a devastating intraocular infection that can result in severe visual morbidity and even loss of the diseased eye if not rapidly and properly managed [1]. The

\section{KARGER}

Kapil G. Kapoor, MD

Wagner Macula and Retina Center

5520 Greenwich Road

Virginia Beach, VA 23462 (USA)

E-Mail Kaps2003@gmail.com 
leading cause of posttraumatic and postoperative endophthalmitis is the colonization with exogenous Gram-positive bacteria that usually dominate the normal ocular surface, with a significant percentage of the causative organisms being Staphylococcus aureus [2, 3]. Postoperative endophthalmitis is fairly rare, but the incidence has increased over the last decade [1]. Increase in drug-resistant organisms is one of the more serious aspects contributing to this increased risk to patients [1].

Traditional management of endophthalmitis includes performing either vitreous tap/biopsy or vitrectomy and delivering appropriate intravitreal antibiotic therapy [3]. Since the Endophthalmitis Vitrectomy Study (EVS) published in 1995, the standard treatment of vancomycin for postoperative endophthalmitis has remained relatively static [4]. However, recent reports of vancomycin resistance in Gram-positive bacteria associated with endophthalmitis and increasing extra-ocular vancomycin resistance have both pointed to the need for alternative treatment tools for Gram-positive intraocular infections [1].

Newer antibiotics designated for vancomycin-resistant infections are commercially available. The preclinical study by Comer et al. [4] uses rabbit models to demonstrate that of these antimicrobials, daptomycin (DAP) appears to offer the greatest solution of providing both a viable treatment alternative and final outcome improvements. There has been one case report on methicillin-resistant $S$. aureus (MRSA) endogenous endophthalmitis treated with intravitreal DAP $(200 \mu \mathrm{g} / 0.1 \mathrm{ml})$ [5]. However, this is the first report to our knowledge of intravitreal DAP used to successfully treat culture-negative recalcitrant exogenous endophthalmitis. Our current report illustrates the multidimensional strategy and flexible approaches required to find the optimal management of postoperative endophthalmitis.

\section{Case Report}

A 63-year-old Caucasian male who had a history of retinal tear in the left eye after successful laser retinopexy 2 years before, subsequently developed an epiretinal membrane that was being monitored. On routine follow-up, he reported increasing metamorphopsia and blurred vision in the left eye that was now interfering with his ability to perform daily occupational tasks. His visual acuity at that visit was 20/30 in the right eye and 20/150 in the left eye. Examination revealed a significant macular pucker in the left eye with macular edema (central macular thickness $=632 \mu \mathrm{m}$ ). Given his significant decrease in vision, the significant progression and contraction of the epiretinal membrane, and the impact on his life, he was offered epiretinal membrane surgery. After a full discussion of risks, benefits, and alternatives, he elected to proceed.

He underwent routine epiretinal membrane peeling under standard sterile precautions without any complications with a 23-guage pars plana sutureless vitrectomy technique. On postoperative day 1 , he was healing well with only trace anterior chamber cell and flare. On postoperative day 3 , he presented with hand motions vision, significant eye pain, a 3-mm hypopyon with 4+ cell/flare, and 3+ corneal edema with no view to the posterior segment. B-scan revealed no retinal detachment. A vitreous tap and injection of intravitreal $0.1 \mathrm{ml}$ vancomycin $/ 0.1 \mathrm{ml}$ ceftazidime/and $0.1 \mathrm{ml}$ of dexamethasone was done. He was started on oral Avelox $400 \mathrm{mg}$ /day, topical atropine, and topical prednisolone. The Gram stain revealed rare Gram-positive cocci, but the culture was negative. On postoperative day 5, the infection progressed with increased hypopyon and increased fibrin. The decision was made to proceed with anterior chamber washout and core vitrectomy with repeat antibiotics (vancomycin and ceftazidime) and culture. 
Sim et al.: Intravitreal Daptomycin for Recalcitrant Postoperative Endophthalmitis

The culture was again negative. On postoperative day 1 following this surgery, his anterior segment was clear of hypopyon with $1+$ cell/flare, and there was some residual peripheral vitreous debris. On postoperative day 3, he redeveloped a 3-mm hypopyon and underwent repeat intravitreal injections of vancomycin and ceftazidime. On postoperative day 4 , his hypopyon height progressed, pain increased, and corneal edema worsened. The decision was made to undergo repeat pars plana vitrectomy, and anterior chamber washout with injections of intravitreal DAP $(200 \mu \mathrm{g} / 0.1 \mathrm{ml})$ [5]. This time postoperatively his eye was quiet with only trace cell/flare and no hypopyon. His eye remained quiet through his postoperative course. There was no evidence of retinal toxicity from DAP. His ultimate vision was counting fingers, attributed to the recalcitrance of his initial infection. His topical prednisolone drops were gradually tapered over 8 weeks with no recurrence of intraocular inflammation or infection.

\section{Conclusion}

The patient's history and course suggest that he developed Gram-positive bacterial endophthalmitis that was fastidious to grow on culture and was likely resistant to vancomycin. Only the intravitreal DAP succeeded in treating his recalcitrant infection. This case study suggests that vancomycin resistance should be considered in culture-negative endophthalmitis, and DAP should be considered as an important treatment alternative.

Vancomycin is a widely used glycopeptide antibiotic that clinicians have traditionally used intravitreously against Gram-positive organisms because of its broad-spectrum activity, minimal resistance, acceptable rates of intraocular sterilization, and lack of toxicity [4]. It is also commonly used to treat the increasing cases of MRSA endophthalmitis [3]. Vancomycin resistant mutants are very rare, but several recent publications have reported increasing resistance to vancomycin in extraocular infections caused by common Gram-positive endophthalmitis isolates such as Staphylococcus, Streptococcus, and Enterococcus sp. [4].

Although vancomycin-resistant endophthalmitis is limited to a few case reports [5-9], the appearance of endophthalmitis caused by vancomycin-resistant enterococci (VRE) and vancomycin-resistant $S$. aureus (VRSA) accelerated shortly after the MRSA endemic emerged in medical centers in the 1990s. VRE endophthalmitis has been described in 4 patients to date: a hospitalized immunocompromised patient with bacteremia, an immunocompetent patient after keratoplasty with infected donor tissue, an immunocompetent patient 20 years following trabeculectomy, and an immunocompetent patient following cataracts surgery [7]. Furthermore, vancomycin resistance in endophthalmitis was commonly noted in Bacillus sp., followed by Staphylococcus sp., and then Enterococcus sp. [3].

The current case report suggests that vancomycin resistance is not only confined to Gram-positive or culture-positive endophthalmitis, but that vancomycin resistance should be considered in culture-negative endophthalmitis. In the EVS, culture-negative or cultureequivocal cases were common at 17.9 and $12.9 \%$, respectively, totaling $30.8 \%$ of cases [10]. Chakrabarti et al. [10] further confirmed that studies of endophthalmitis must presume a $25-30 \%$ rate of culture-negative endophthalmitis. This case proposes that we ought to consider vancomycin resistance as a possibility in these $30 \%$ of endophthalmitis cases. Finetuning our clinical roadmap in these cases will find increasing importance in the setting of rising resistance rates, particularly since cases of vancomycin-resistant endophthalmitis are often associated with worse visual outcomes [3].

DAP is a lipopeptide antibiotic that is approved to treat skin and soft tissue infections, bacteremia, endocarditis, and osteomyelitis $[2,5]$. Although DAP is not formally recom- 
Sim et al.: Intravitreal Daptomycin for Recalcitrant Postoperative Endophthalmitis

mended for endophthalmitis treatment, it has shown to have a significantly longer half-life than vancomycin, and to be just as effective as vancomycin [2]. A 1-mg dose of DAP ensures noninferiority from the bactericidal efficacy of a 1-mg dose of vancomycin [2]. DAP also demonstrates superior in vitro activity for vancomycin-resistant bacteria strains typically accounting for endophthalmitis [4]. These strains include: S. epidermidis, S. aureus, S. pneumoniae, E. faecalis, and E. faecium [4]. DAP kills $99.9 \%$ of Gram-positive bacteria within 6-8 h compared to 12-24 h or simple bacteriostasis with vancomycin [4]. In a rabbit ventriculitis model, DAP achieved superior bactericidal activity and more rapid kill-times; antibacterial activity of DAP is rapid and concentration dependent, while antibacterial activity of vancomycin is slow and time dependent $[2,4]$.

Comer et al. [4] also demonstrated that administration of $200 \mu \mathrm{g}$ of intravitreal DAP was safe and efficacious in a rabbit model of bacterial endophthalmitis. Higher concentrations lead to a dose-dependent increase in cataract formation, ERG suppression, and photoreceptor damage [4]. The therapeutic window may seem narrow, but $200 \mu \mathrm{g}$ of intravitreal DAP resulted in near complete vitreous sterilization $24 \mathrm{~h}$ after treatment, and complete vitreous sterilization by $48 \mathrm{~h}$ [4]. Moreover, clinical and histopathologic examinations confirmed preservation of the retina, photoreceptor layers, ocular structure architecture, and limited ocular inflammation after sterilization of the treated eye $[2,4]$.

In conclusion, vancomycin resistance among Gram-positive isolates is an uncommon, but emerging challenge. The importance of antibiotic resistance requires ongoing surveillance and periodic reporting from individual laboratories. Vancomycin resistance should be considered in culture-negative endophthalmitis recalcitrant to treatment, and DAP should be considered as an alternative in these cases, though further studies will be needed to better determine its therapeutic utility in human clinical trials.

\section{Acknowledgment}

This case report was funded by Eastern Virginia Medical School Office of Research.

\section{Statement of Ethics}

The patient gave informed consent and the study protocol adhered to the ethical guidelines of the institute's committee on human research.

\section{Disclosure Statement}

The authors have no disclosures. 
Sim et al.: Intravitreal Daptomycin for Recalcitrant Postoperative Endophthalmitis

\section{References}

1 Maguire JL: Postoperative endophthalmitis: optimal management and the role and timing of vitrectomy surgery. Eye 2008;22:1290-1300.

-2 Lefevre S, Saleh M, Marcellin L, et al: Daptomycin versus vancomycin in a methicillin-resistant Staphylococcus aureus endophthalmitis rabbit model: bactericidal effect, safety, and ocular pharmacokinetics. Antimicrob Agents Chemotherapy 2012;56:2485-2492.

-3 Khera M, Avinash P, Animesh J, et al: Vancomycin-resistant Gram-positive bacterial endophthalmitis: epidemiology, treatment options, and outcomes. J Ophthalmic Inflamm Infect 2013;3:46.

-4 Comer GM, Miller JB, Schneider EW, et al: Intravitreal daptomycin: a safety and efficacy study. Retina 2011;31:1199-1206.

5 Buzzacco DM, Carroll PC: Intravitreal daptomycin in a case of bilateral endogenous endophthalmitis. Arch Ophthalmol 2012;130:940.

-6 Bains HS, Weinberg DV, Feder RS, Noskin GA: Postoperative vancomycin-resistant Enterococcus faecium rndophthalmitis. Arch Ophthalmol 2007;125:1292-1293.

7 Sharma S, Desai RU, Pass AB, Saffra NA: Vancomycin-resistant Enterococcal endophthalmitis. Arch Ophthalmol 2010;128:794-795.

$>8$ Esmaeli B, Holz E, Ahmadi A, et al: Endogenous endophthalmitis secondary to vancomycin-resistant Enterococci infection. Retina 2003;23:118-119.

-9 Tang CW, Cheng CK, Lee TS: Community-acquired bleb-related endophthalmitis caused by vancomycinresistant enterococci. Can J Ophthalmol 2007;42:477-478.

10 Chakrabarti M, John SR, Chakrabarti A: Culture negative (sterile) postoperative endophthalmitis. Kerala J Ophthalmol 2009;21:190-193. 